\title{
Rivastigmine for the treatment of dementia associated with Parkinson's disease
}

\author{
Jennifer L Reingold \\ John C Morgan \\ Kapil D Sethi
}

Movement Disorders Program, Department of Neurology, Medical College of Georgia, Augusta, GA, USA

\begin{abstract}
Parkinson's disease (PD) afflicts millions of people worldwide and leads to cognitive impairment or dementia in the majority of patients over time. Parkinson's disease dementia (PDD) is characterized by deficits in attention, executive and visuospatial function, and memory. The clinical diagnostic criteria and neuropathology surrounding PDD remain controversial with evidence of overlap among PDD, dementia with Lewy bodies (DLB) and Alzheimer's disease (AD). Cortical cholinergic deficits are greater in PDD than in AD, and are well-correlated with the cognitive and neuropsychiatric dysfunction that occurs in PDD. Inhibition of acetylcholine metabolism is therefore a practical therapeutic strategy in PDD.

This review examines current evidence for rivastigmine (a cholinesterase/butyrylcholinesterase inhibitor) treatment in PDD. In addition to its efficacy, we examine the safety profile, side effects, and cost effectiveness of rivastigmine in PDD. Rivastigmine provides modest benefit in PDD and further long-term studies are needed to determine the effectiveness and safety of rivastigmine over time. Tolerability is a problem for many PDD patients treated with rivastigmine. Future studies of rivastigmine in PDD should focus on pragmatic outcomes such as time to need for nursing home placement, pharmacoeconomic outcomes and simultaneous patient/caregiver quality of life assessments.
\end{abstract}

Keywords: Parkinson's disease, dementia, rivastigmine, cholinesterase inhibitor

\section{Introduction}

Parkinson's disease (PD) is the second most common neurodegenerative disease behind Alzheimer's disease (AD). James Parkinson (1817) first described PD in his seminal work "An Essay on the Shaking Palsy." The cardinal motor signs of PD (bradykinesia, resting tremor, cogwheel rigidity, postural instability) are emphasized in making the diagnosis and in tracking progression of the disease (Gibb and Lees 1988). Most recently, significant attention has been given to the non-motor symptoms of PD, including constipation, depression, olfactory dysfunction and dementia (Chaudhuri et al 2006). Parkinson (1817) was keenly aware of many non-motor aspects of PD including constipation and disturbed sleep. He did not recognize, however, impaired olfaction and dementia as a part of the disease describing "the senses and intellects" as "being uninjured" (Parkinson 1817). His lack of recognition of PD as a dementing illness is understandable given life expectancy in Great Britain in the early 19th century was under 40-years, and we now know that the risk of Parkinson's disease dementia (PDD) increases with age (Levy et al 2002).

PDD has perhaps been more studied and emphasized of late for several reasons: (1) greater emphasis on the non-motor symptoms of PD (Chaudhuri et al 2006), (2) the emergence of cholinesterase inhibitors as effective treatments in AD and PDD, and (3) ruling-out dementia is important in PD patients being considered for deep brain stimulation surgery (given it is exclusionary). We do know that PDD is under recognized and under treated in routine clinical practice. Recent studies have demonstrated that cognitive decline and/or PDD afflicts the majority of patients with PD 
over time (Aarsland et al 2003; Hely et al 2005) and that this significantly contributes to increased morbidity and mortality (Levy et al 2002; Hughes et al 2004; de Lau et al 2005).

\section{Defining Parkinson's disease dementia (PDD)}

According to DSM-IV criteria (APA 2000), dementia is characterized by "the development of multiple cognitive deficits that include memory impairment and at least one of the following cognitive disturbances: aphasia, apraxia, agnosia, or disturbance in executive dysfunction. The cognitive deficits must be sufficiently severe to cause impairment in occupational or social functioning and must represent a decline from a previously higher level of functioning." Major cognitive domains that can be affected in dementia include: (1) executive function, (2) recent memory, (3) language, and (4) visuospatial function. PDD is more of a subcortical dementia with prominent deficits in executive and visuospatial function typically more so than language and recent memory (Cummings 1988; Rippon and Marder 2005). PDD is also characterized by generalized cognitive slowing (bradyphrenia) and impaired attention (Cummings 1988; Rippon and Marder 2005).

Mild cognitive impairment (MCI) is differentiated from dementia in that it represents a borderland between normal cognition and dementia. MCI remains controversial with some clinicians viewing this entity as a disease along a pathway leading to fulminant $\mathrm{AD}$, while others view MCI as a heterogeneous syndrome representing an early stage of different forms of dementia (Fernandez et al 2005). Cognitive decline is perhaps the rule as PD progresses (Hely et al 2005); however, the definition of MCI in PD is even less well-defined than the definition of PDD (Fernandez et al 2005).

Given clinical and neuropathological overlap, the most important distinction in the diagnosis of PDD is attempting to distinguish it from dementia with Lewy bodies (DLB) (Aarsland et al 2005; McKeith et al 2005). Clinically, patients with DLB are typically characterized as having early dementia and parkinsonism with less robust response to levodopa, fluctuations in level of alertness, visual hallucinations in the absence of dopaminergic treatment, rapid progression of dementia, and severe sensitivity to typical and some atypical antipsychotics (Fuchs et al 2004; McKeith et al 2005). Unfortunately, neuropsychological testing may not be able to distinguish between patients with these two entities (Noe et al 2004), and the timecourse of the development of the dementia is perhaps most important.
For an operational method of distinguishing PDD from DLB, PDD has recently been defined as the onset of dementia at least one year after the appearance of Parkinsonian motor symptoms (McKeith et al 2005). This definition is artificial and not typically representative of clinical experience (where patients become demented after many years of PD (Aarsland et al 2005)) and maybe difficult to apply in practice. The largest clinical trial in PDD (Emre et al 2004) included patients meeting established clinical diagnostic criteria for PD (Gibb and Lees 1988) and fulfilling DSM-IV criteria for PDD with onset at least two years after the diagnosis of PD (Emre et al 2004).

Neuropathologically PDD overlaps with DLB and AD in numerous studies (Mahler and Cummings 1990; Perl et al 1998; Aarsland et al 2004; Galvin et al 2006). Dementia with Lewy Bodies (DLB) and PDD are typically categorized as two separate disease states with distinct pathologies, but debate continues as to whether DLB and PDD are, in fact, along the same disease spectrum (Levy et al 2006; Galvin et al 2006; Padovani et al 2006). AD pathology has been noted in many demented and non-demented patients with PD and estimates of AD pathology among PD patients at autopsy are as high as $42 \%-91 \%$ (Galvin et al 2006; Padovani et al 2006). Although these changes are present in a large proportion of patients with PD, it is difficult to define the role these changes play in the cognitive decline in PD.

It is also suggested that the cognitive dysfunction developing later in Parkinson's is due to the loss of cholinergic neurons in the nucleus basalis of Meynert (Whitehouse et al 1987). Supporting this hypothesis, there is evidence that cortical cholinergic deficits are more pronounced in PD than in $\mathrm{AD}$ and well-correlated with cognitive decline and neuropsychiatric disturbances in PD (Bohnen et al 2003; Hilker et al 2005).

Most recently, Aarsland et al (2005) reported the neuropathology of $22 \mathrm{PD}$ patients identified in the community and followed prospectively until death. Eighteen of the 22 patients (82\%!!) were diagnosed with dementia with none of the 18 meeting established neuropathological criteria for AD (Aarsland et al 2005). All 18 demented patients had limbic and neocortical Lewy bodies (Aarsland et al 2005) however. In accord with clinical practice, the average disease duration for these patients was approximately 16 years with a diagnosis of dementia occurring a mean of three years before death (Aarsland et al 2005). It thus appears that limbic and neocortical Lewy bodies associated with significant cholinergic deficits are perhaps the main substrate for the development of PDD. 


\section{Risk factors and epidemiology of PDD}

The Apo $\varepsilon 2$ allele, older age at onset, the presence of depression, development of visual hallucinations, severity of disease, and an akinetic-rigid phenotype are established risk factors for the development of PDD (Marder et al 1995; Levy et al 2002; de Lau et al 2005; Burn et al 2006). The incidence and prevalence of PDD depends upon the neuropsychological tests and the criteria used to define PDD and neuropsychological testing batteries can vary considerably from center to center (Defer et al 1999; Saint-Cyr and Trepanier 2000; Pillon 2002; Burn et al 2006; Voon et al 2006).

The incidence of PDD varies widely across studies with the greatest incidence in elderly PD patients. The risk of developing dementia in PD is estimated to be 6 times that of age-matched controls (Padovani et al 2006). Early estimates of the prevalence of dementia in PD were in the range of $20 \%$ (Brown and Marsden 1984). Cummings (1988) estimated that as many as 40 percent of PD patients cross-sectionally have PDD. More recently, Aarsland et al (2003) found that 78\% of PD patients followed for 8 years develop PDD (Aarsland et al 2003).

The prevalence of MCI in PD is largely unknown (Fernandez et al 2005). Cognitive decline, perhaps consistent with MCI, was present in $84 \%$ of survivors from a cohort of PD patients followed 15-years after diagnosis (Hely et al 2005). Forty-eight percent of these survivors fulfilled criteria for dementia (Hely et al 2005). Consistent with these findings, most PD patients undergoing neuropsychological testing to exclude dementia before deep brain stimulation surgery display some level of cognitive dysfunction (typically executive dysfunction) (Saint-Cyr et al 2000; Pillon 2002). Cognitive decline is therefore perhaps a ubiquitous feature as PD progresses.

\section{Rivastigmine}

Acetylcholinesterase (AChE) inhibitors have been the mainstay of treatment in $\mathrm{AD}$ for many years. In $\mathrm{AD}$, these drugs have been shown to slow symptomatic decline in cognition over time, delay nursing home placement, and result in lower long-term healthcare costs for patients/families (Geldmacher 2005). AChE inhibitors theoretically improve cognitive function by increasing acetylcholine in the brain through preventing its breakdown. There are numerous AChE inhibitors commercially available including donepezil, galantamine, rivastigmine, and tacrine. All of these drugs are effective in $\mathrm{AD}$, however there is relatively little data on their use in PDD.

Rivastigmine is a second generation, carbamate-type, reversible, brain selective cholinesterase inhibitor (Enz et al 1991). It appears to be more selective for the monomeric form of $\mathrm{AChE}$ that is predominantly in the cortex and hippocampus relative to peripheral forms of $\mathrm{AChE}$ (Enz et al 1993). It is dosed twice daily titrating from $1.5 \mathrm{mg}$ twice per day up to $6 \mathrm{mg}$ twice per day as tolerated. The majority of patients tolerate total doses of $6 \mathrm{mg} /$ day or higher. Rivastigmine is slightly different than donepezil and galantamine given it has both $\mathrm{AChE}$ inhibitory activity and butyrylcholinesterase inhibitory activity. Inhibition of both forms of cholinesterase has theoretical advantages in allowing greater endogenous levels of acetylcholine, but it is unknown if this drug provides benefits above and beyond other AChE inhibitors given headto-head studies have not been done.

\section{Clinical trials of rivastigmine in DLB and PDD}

Donepezil and galantamine have only been studied in open-label studies or small scale clinical trials in

Table I Placebo-controlled cholinesterase inhibitor trials in PDD and DLB

\begin{tabular}{|c|c|c|c|c|c|}
\hline Authors & Medication & Study design & Number of patients & Study duration & $\begin{array}{l}\text { Diagnostic criteria for } \\
\text { PDD/DLB }\end{array}$ \\
\hline McKeith et al (2000) & Rivastigmine & $\mathrm{DB}, \mathrm{PC}$ & 120 & 23 weeks & $\begin{array}{l}\text { Probable DLB, mild to } \\
\text { moderate dementia } \\
\text { with MMSE >9 }\end{array}$ \\
\hline Aarsland et al (2002) & Donepezil & $\mathrm{DB}, \mathrm{PC}, \mathrm{CO}$ & 14 & 10 weeks & DSM-IV/probable PDD \\
\hline Leroi et al (2004) & Donepezil & $\mathrm{DB}, \mathrm{PC}$ & 16 & 18 weeks & $\begin{array}{l}\text { DSM-IV or symptoms } \\
\text { consistent with PDD }\end{array}$ \\
\hline Emre et al (2004) & Rivastigmine & $\mathrm{DB}, \mathrm{PC}$ & 541 & 24 weeks & $\begin{array}{l}\text { DSM-IV, mild to mod- } \\
\text { erate dementia with } \\
\text { MMSE of 10-24 }\end{array}$ \\
\hline Ravina et al (2005) & Donepezil & $\mathrm{DB}, \mathrm{PC}, \mathrm{CO}$ & 22 & 10 weeks & $\begin{array}{l}\text { DSM-IV, mild to mod- } \\
\text { erate dementia with } \\
\text { MMSE I7-26 }\end{array}$ \\
\hline
\end{tabular}

Abbreviations: DB, double-blind; PC, placebo-controlled; CO, cross-over. 
PDD (Table 1). Rivastigmine has been studied in large randomized, placebo-controlled clinical trials in DLB (McKeith et al 2000) and PDD (Emre et al 2004) (Table 1). The data available for rivastigmine has led to the approval of this medication in the treatment of PDD in Europe, the United States and elsewhere.

McKeith et al (2000) studied the efficacy of rivastigmine in DLB. In this randomized, double-blind, placebocontrolled study, participants were given placebo or titrated up to $12 \mathrm{mg} /$ day of rivastigmine for 20 -weeks followed by a 3-week washout period. Assessments were made at baseline, and at 12-, 20-, and 23-weeks. Neuropsychiatric testing and computerized cognitive assessments were performed at each session. Significant clinical and cognitive performance improvements were noted in those receiving rivastigmine as compared with placebo. Thirty-seven subjects (63\%) in the rivastigmine group showed at least a 30 percent improvement from baseline as compared to only 18 subjects $(30 \%)$ of the placebo group. Patients improved most in the areas of apathy and indifference and had fewer hallucinations than those receiving placebo.

As previously discussed, it is becoming more evident that DLB and PDD are on a continuum of disease, with significant overlap in terms of clinical and cognitive signs and symptoms (Aarsland et al 2005; Galvin et al 2006; Padovani et al 2006). Given the obvious clinical benefits of rivastigmine in DLB (McKeith et al 2000), this led to further study in patients defined as having PDD.

In an open label trial by Reading et al (2001), twelve patients with PD-related cognitive impairment and psychosis were given rivastigmine at an initial dose of $1.5 \mathrm{mg}$ twice daily and then titrated to $6 \mathrm{mg}$ twice daily or the highest tolerated dose. Patients were assessed at 8-weeks after the maximum titration level was reached and once again 6 -weeks after that period. The drug was discontinued at that point and participants were assessed once again 3-weeks after withdrawal of rivastigmine. At each session, the Folstein Mini-Mental Status Exam (MMSE) was utilized as a measure of cognition and the Unified Parkinson's Disease Rating Scale (UPDRS) was administered to evaluate the motor symptoms of PD. Neuropsychiatric symptoms were evaluated using the Neuropsychiatric Inventory (NPI), which evaluates behaviors over the preceding four weeks. Cognitive assessment utilizing the MMSE revealed significant improvement of 5 points in patients on rivastigmine relative to their baseline $(Z=2.81, \mathrm{p}<0.005)$. Motor symptoms and signs were unchanged as measured by UDPRS $(Z=1.18, \mathrm{p}>0.2)$. NPI scores were also significantly lowered (improved) from baseline on treatment $(Z=2.85, p<0.004)$. Patients worsened significantly three weeks after withdrawal of the rivastigmine (Reading et al 2001).

Giladi et al (2003) also studied the efficacy of rivastigmine in the treatment of PDD. In this open label study, 20 of 28 patients completed 26-weeks of treatment with rivastigmine therapy (as tolerated up to $12 \mathrm{mg} /$ day). The MMSE and Alzheimer's Disease Assessment Scale (ADAS-cog) were used as cognitive measures and the UPDRS for documenting Parkinsonian features. Patients were assessed prior to starting therapy, at 12 and 26-weeks while on rivastigmine, and 8 -weeks after withdrawal of therapy. Eight of the 28 patients dropped out secondary to side effects. An increase in UDPRS score was observed from baseline to week $26(\mathrm{p}>0.06)$ and a non-significant improvement was noted from week 26 to 8-weeks after treatment washout. Significant improvement was noted in the area of the attentional components of the MMSE at week $26(\mathrm{p}<0.002)$. In terms of the ADAS-cog, a significant increase in total score (worsening) was noted throughout the study period $(\mathrm{p}=0.002)$.

The authors indicated that the objective cognitive measures (MMSE and ADAS-cog) did not at all reflect the clinical impressions of the caregivers for the patients in the study. In particular, caregivers seemed surprise by the deterioration experienced after washout. This, again, suggests the need for a cognition assessment tool that is validated and best utilized in PD. Giladi et al (2003) also noted increased tremor in eleven of the original 28 participants in the study and dose reduction was required.

In this study, it would be expected for patients to worsen on the UPDRS over 6-months whether they were on an AChE inhibitor or not. A non-significant trend for improvement in the UPDRS scores following washout, however would make one consider that rivastigmine negatively influenced motor performance. An increase in tremor and parkinsonism was a legitimate concern in this study given we still use anticholinergics for some patients with PD (certainly not demented ones, though!) (Morgan and Sethi 2005). There were prior case reports of worsening of tremor and parkinsonism in PD patients treated with AChE (Richard et al 2002). The results of the Reading et al (2001) and Giladi et al (2003) studies indicated further need to study the effects of this drug on cognition and parkinsonism in PDD in a prospective, doubleblind, placebo-controlled fashion.

In 2003, Fogelson et al (2003) performed another open label study examining the effects of rivastigmine on quantitative EEG (qEEG) in PDD patients $(n=19)$, given they frequently have a slowing of alpha activity on EEG. Patients 
were treated with rivastigmine at an initial dose of $3 \mathrm{mg} /$ day and titrated to a dose of $12 \mathrm{mg} /$ day or highest tolerated dosage. Quantitative EEG recordings were performed prior to introduction of rivastigmine and repeated when the patients had been on treatment for 12-weeks. A significant increase in the relative alpha activity was noted after treatment with rivastigmine ( $\mathrm{p}=0.019)$, however, no correlation between qEEG changes and cognitive improvement was identified (Fogelson et al 2003). It is difficult, therefore, to determine whether these qEEG changes were due to improvement in cognitive state rather than just an increase in arousal.

A sound neuropathological and pharmacological basis and promising open label studies were followed by the publication of a multi-center, placebo-controlled, double-blind study in 541 patients with PDD (Emre et al 2004). PD was diagnosed using the UK PD Brain Bank Criteria and dementia was diagnosed using DSM-IV criteria. Participants were randomly assigned to receive placebo or 3-12 $\mathrm{mg}$ (titrated to the maximum tolerated dose over a 16-week dose escalation period) of rivastigmine divided twice daily for 24-weeks. Patients were randomized 2:1 to rivastigmine and placebo, respectively. The primary outcome measures were the ADAS-cog scores as in previous open label studies (Fogelson et al 2003; Giladi et al 2003) and the Alzheimer's Disease Cooperative Study-Clinician's Global Impression of Change (ADCS-CGIC). There were six secondary outcome measures: Alzheimer's Disease Cooperative Study - Activities of Daily Living (ADCS-ADL), NPI, MMSE, Cognitive Drug Research Power of Attention tests (CDR), Delis-Kaplan Executive Function System (D-KEFS) verbal fluency test, and the Ten Point Clock-Drawing Test. Safety monitoring included recording of adverse events, monitoring ECGs and laboratory data, vital signs and body weight. Patients were also assessed for changes in parkinsonism from baseline scores at weeks 16 and 24 using the UPDRS part III (motor section) score.

Patients were included in the efficacy analysis if they underwent a baseline evaluation and if they took one dose of study medication followed by an assessment of the one of the efficacy variables after baseline (regardless if they were taking study medication at the time). If no followup information was available Emre et al (2004) used the last-observation-carried-forward method to impute missing values for missing follow-up information.

Approximately $30 \%-35 \%$ of patients enrolled in the study suffered with co-morbid psychiatric disorders (including depression, anxiety, and psychosis) (Emre et al 2004). The average age of patients was approximately 72 with PD diagnosis 9-years earlier on average. Essentially all of the patients were Caucasian and approximately $2 / 3$ were men. The mean time since the diagnosis of PDD for patients entering the study was approximately $13-15$ months. There were no significant demographic differences between the two treatment groups.

As compared to patients in the placebo group, patients who received rivastigmine demonstrated significant improvements in ADAS-cog and ADCS-CGIC scores (primary efficacy variables) (Emre et al 2004). Patients that were treated with rivastigmine had a mean improvement of 2.1 points in the ADAS-cog, while patients in the placebo group had a 0.7 point worsening $(p, 0.001)$. Clinically meaningful improvement was observed in the investigator rated ADS-CGIC in $19.8 \%$ or rivastigmine patients and $14.5 \%$ in placebo-treated patients, while clinically meaningful worsening was evident in $13.0 \%$ and 23.1 percent, respectively $(\mathrm{p}=0.007)$. At week 24 , rivastigmine provided improvement in all six secondary efficacy variables relative to the baseline evaluation, while placebo-treated patients remained the same (NPI) or worsened (ADCS-ADL, MMSE, CDR, D-KEFS, Ten Point Clock-Drawing Test) (Emre et al 2004). These benefits should be considered moderate and in-line with other clinical trials of AChE inhibitors in AD.

A total of 410 out of 541 patients enrolled completed the study. Ninety-nine patients dropped out of the study in the rivastigmine arm with 32 dropping-out in the placebo arm. Approximately two-thirds of the drop-outs in the rivastigmine arm were due to adverse events and less than half of the drop-outs in the placebo arm were due to the same. Cholinergic symptoms typical of AChE inhibitors were the most common adverse events, with nausea reported by $29 \%$ of rivastigmine - vs $11.2 \%$ of placebo-treated patients ( $p<0.001$ ) and vomiting by $16.6 \%$ vs $1.7 \%$ respectively. In general, Parkinsonian symptoms as a whole were more often reported by patients in the rivastigmine group relative to placebo $(27.3 \%$ vs $15.6 \%, \mathrm{p}=0.002)$. Tremor $(10.2 \%$ vs $3.9 \%$ ) and dizziness ( $5.8 \%$ vs $1.1 \%$ ) were also reported more often as an adverse event in rivastigmine-treated patients. Tremor only caused withdrawal of $1.7 \%$ of patients in the rivastigmine group and no one in the placebo group $(\mathrm{p}=0.19)$. There was no significant difference in UPDRS motor scores and tremor related items between the groups, however. Interestingly, hallucinations (4.7\% vs 9.5\%) and orthostatic hypotension ( $1.7 \%$ vs $5.0 \%$ ) were reported more often in patients treated with placebo.

In a letter to the editor, Harada et al (2005) argued that the number needed to treat in order to reach what Emre et al 
(2004) defined as "clinically meaningful improvement" does not outweigh the side effects experienced by the participants in the study. Harada et al (2005) only considered those who had "clinically meaningful benefit" in their analysis, however, and an important factor in any progressive neurodegenerative dementia is how many patients were prevented from "clinically meaningful worsening" as well.

The results of the Emre et al (2004) study are encouraging for the use of rivastigmine in PDD, however the efficacy is modest. Adverse events are common with rivastigmine and typically cholinergic in nature, however they usually do not result in discontinuation of the drug. This study was hampered by lack of a validated assessment tool for PDD. The ADAS-cog and the ADCS-CGIC as primary efficacy variables are appropriate, however there needs to be further validation of this scale in PDD. Fortunately, six additional tests of cognitive domains that would be impaired in PDD were also used (CDR, etc.). The finding of fewer reported hallucinations in the rivastigmine-treated arm is interesting given this drug may not only provide stabilization and slower decline of cognitive function in PDD, it may also help reduce hallucinations, which can be quite troubling for patients and caregivers alike.

Another concern is the cost of this drug relative to the benefit. Is the modest benefit obtained enough to delay nursing home placement and is it cost-effective over time? Should NMDA-receptor antagonists such as memantine be added to PDD as it advances? Are the other AChE inhibitors equally efficacious in PDD?

There was an open-label extension to the Emre et al (2004) study published recently by Poewe et al (2006). Of 433 patients that completed the double-blind trial, 334 entered and 273 completed the active treatment extension study (3-12 mg rivastigmine/day). At 48-weeks the ADAS-cog score had improved by 2 points above baseline for the entire group of patients. Patients in the placebo treatment arm in the original Emre et al (2004) trial also had a 2-point improvement in their ADAS-cog score. The safety profile of rivastigmine in the open label extension was similar to the double-blind phase (Poewe et al 2006).

Wesnes et al (2005) looked specifically at the effects of rivastigmine on attention in PDD in patient enrolled in the Emre et al (2004) trial. In this sub-study, 487 patients with PDD were given rivastigmine or placebo and assessed at baseline, 16 and 24-weeks. Assessment of attention on the Cognitive Drug Research (CDR) computerized assessment was performed at each visit in order to assess attention during various tasks. As compared with placebo, significant benefits on attention were noted among the rivastigmine group. Difficulty maintaining attention is a common finding in PDD and further asserts that rivastigmine is helpful for this impairment in these patients (Wesnes et al 2005).

Economic evaluation of rivastigmine was examined soon after positive studies on PDD were published. Willan et al (2006) prospectively examined the cost effectiveness of rivastigmine in the Emre et al (2004) treated patients. Quality adjusted survival time (QAST) score was transformed from the MMSE score and utilized as a measure of cost effectiveness. Although an increase in QAST in the rivastigmine arm of 2.81 quality-adjusted life-days was noted (two-sided p-value 0.13 [ $90 \%$ CI $-0.243,5.86]$ ), no between-treatment differences in cost were seen. The high variability in cost of medications (the study looked at Canadian and UK prices) as well as the short duration of the study (six months) could have interfered with the examination of cost effectiveness and further studies need to be performed.

Patients with PDD and DLB can often have autonomic nervous system dysfunction, and $\mathrm{AChE}$ can potentially increase acetylcholine and contribute to cardiac dysfunction. Ballard et al (2006) reviewed cardiac safety of rivastigmine in DLB and PDD. Reviewing the Emre et al (2004) PDD trial ( $n=541)$ and the McKeith et al (2000) DLB trial $(n=120)$, no clinically meaningful treatment differences in bradycardia or abnormalities on ECG were noted. Patients treated with rivastigmine did have a mean reduction of 1.5-2 beats per minute in heart rate, however. In fact, compared with placebo, it appears that rivastigmine was associated with fewer adverse events $(p=0.002)$ and fewer syncopal episodes $(p=0.018)$ among PDD patients (Ballard et al 2006).

\section{Discussion}

A recent practice parameter from the American Academy of Neurology indicated that there was Level B evidence (moderately strong) for the treatment of PDD with either donepezil or rivastigmine (Miyasaki et al 2006). Given the lack of published large, randomized, placebo-controlled trials of donepezil in PDD, we feel that rivastigmine currently has the best data for use in PDD. The benefits are modest and further studies are needed, but the Emre et al (2004) data are the best that we have in the use of AChE inhibitors in PDD and the clinically and pathologically similar condition DLB (McKeith et al 2002). The dropout rate in the placebocontrolled phase was higher in the rivastigmine-treated PDD patients vs placebo-treated patients (27.3\% vs $17.9 \%)$ (Emre et al 2004) and using LOCF analytical techniques in a progressive condition like PDD would artifactually 
show less worsening in the rivastigmine treated group. The number needed to treat to provide a clinically meaningful significant outcome in the Emre et al (2004) trial is perhaps six if you consider both meaningful improvement in the rivastigmine arm combined with the group with clinically meaningful worsening in the placebo-treated arm (Aarsland et al 2006).

Given the relatively high drop-out rate in rivastigminetreated patients, the safety profile of rivastigmine should be further examined. Future studies need to specifically explore the gastrointestinal side effects and patient perceived worsening of motor symptoms (ie, especially tremor). To this end, a recent study of 26 patients with PDD on rivastigmine revealed only mild worsening of tremor by accelerometry and a global tremor rating scale based upon the UPDRS (Gurevich et al 2006). Further study of the possible positive influence of rivastigmine on the neuropsychiatric symptoms of PDD (hallucinations and delusions) is warranted given these problems were reported significantly less as adverse events in the rivastigmine arm of the Emre et al (2004) placebo-controlled study. It will be interesting to see if the other AChE inhibitors like donepezil (a drug with fewer reported adverse events in AD trials (Birks 2006)) provide similar efficacy in PDD with fewer side effects (Aarsland et al 2002; Leroi et al 2004; Ravina et al 2005).

It is also necessary to look at the cost of rivastigmine versus the benefits gained by patients and caretakers. While there is good pharmacoeconomic data in $\mathrm{AD}$ for various AChE inhibitors (Geldmacher 2005), it is lacking for these drugs in PDD. A pharmacoeconomic assessment performed by Willan et al (2006) was possibly not significant because of the variability of drug costs and the short, six-month study period. Alternatively, the costs may outweigh the benefits of rivastigmine in PDD. Looking ahead, we must examine costs over a longer treatment horizon. A confounding factor in PDD is that progression and severity of motor symptoms can lead to long-term care in patients with PD, which is somewhat different than in $\mathrm{AD}$ where patients are typically put in a nursing home due to severe dementia.

The publication of the efficacy of rivastigmine in PDD could be regarded as "the carriage coming before the horse" so to speak. In our opinion, a well-defined, clinically and neuropathologically relevant definition of PDD must be developed. The Movement Disorders Society has assembled a task force to develop this definition and the outline of this new definition was presented at the 10th International Conference of Parkinson's Disease and Movement Disorders in Kyoto, Japan in 2006. This definition of PDD will hopefully be published in its entirety soon. While a decline in premorbid function will be necessary for the diagnosis of PDD with impairment in at least two cognitive domains, it appears that memory impairment on the MMSE (score < 25) will not be a required feature to make the diagnosis of PDD by these criteria. The current definition of PDD is largely based on the definition of dementia as cited in the DSM-IV with a decline in functional level, memory decline and at least one additional form of cognitive impairment (executive dysfunction, apraxia, agnosia, aphasia) (Fuchs et al 2004) and by exclusion of PDD from those with DLB (McKeith et al 2005).

Screening and assessment tools for MCI in PD patients and in PDD must be identified, developed and validated. If you examine various neuropsychological testing batteries recommended for evaluation of cognitive function in $\mathrm{PD}$, you will find a hodge-podge of cognitive scales and tests with many lacking widespread use and validation in PD (Defer et al 1999; Saint-Cyr et al 2000; Pillon et al 2002; Burn et al 2006; Voon et al 2006). Emre et al (2004), Giladi et al (2003), Fogelson et al (2003), and Reading et al (2002) used cognitive scales that were assessed and validated in AD (ADCS-GCIS, ADCS-ADL, NPI) without significant validation in PDD. Miyasaki et al (2006) identified the most accurate screening tools for PDD as the MMSE and the Cambridge Cognitive Examination (CAMcog). Both tools had similar sensitivities for the diagnosis of PDD; however, the CAMcog (94\%) was more specific than the MMSE (77\%) (Miyasaki et al 2006).

A more precise definition and characterization of mild cognitive impairment (MCI) in PD is also warranted (Fernandez et al 2005). We know that PD is a progressive illness and patients with MCI and without PD typically go on to develop $\mathrm{AD}$ over time. In patients with $\mathrm{PD}$, how many patients have MCI and how many of these go on to develop PDD over time? There is much additional work that needs to be done in these areas to characterize and understand the course our patients will take as they progress.

The Montreal Cognitive Assessment (MoCA) has recently entered into the literature as an assessment tool for MCI. Unlike the MMSE, the MoCA incorporates 8 additional cognitive domains including more complex visuospatial evaluation, naming, attention, memory, language and abstraction tasks. It appears sensitive to the kind of deficits that are evident in PDD (executive and visuospatial dysfunction, etc.). In a validation study performed by Nasreddine et al (2005), the MoCA was found to have high sensitivity and specificity for detecting MCI in patients 
who performed in normal ranges on the MMSE. Using a normal cut-off score of 26/30, the MMSE had a sensitivity of $18 \%$ to detect MCI whereas an abnormal MoCA score detected $90 \%$. While the MoCA seems to have much promise in detection of MCI, further validation in PD and PDD is necessary.

The publication of the first large-scale, randomized, double-blind controlled trial of rivastigmine in PDD has generated much interest in the definition, recognition, and treatment of PDD. While the clinical benefits of rivastigmine for PDD patients and their families appear moderate, and drop-out rates due to adverse events is a concern, this drug shows promise in an under recognized and under treated condition. Rivastigmine provides clinically meaningful benefits in cognition and activities of daily living for some PDD patients in the short-term (Emre et al 2004; Poewe et al 2006); however, future studies are necessary to validate its use in the long-term treatment of PDD.

\section{References}

Aarsland D, Anderson K, Larsen JP, et al. 2003. Prevalence and characteristics of dementia in Parkinson disease: an 8-year prospective study. Arch Neurol, 60:387-92.

Aarsland D, Ballard CG, Halliday G. 2004. Are Parkinson's disease with dementia and dementia with Lewy bodies the same entity? J Geriatr Psychiatry Neurol, 17:137-45.

Aarsland D, Perry R, Brown A, et al. 2005. Neuropathology of dementia in Parkinson's disease: a prospective, community-based study. Ann Neurol, 58:773-6.

[APA] American Psychiatric Association. 2000. Diagnostic and statistical manual of mental disorders (4th edn). Washington: APA.

Ballard C, Lane R, Barone P, et al. 2006. Cardiac safety of rivastigmine in Lewy body and Parkinson's disease dementias. Int J Clin Pract, 60:639-45.

Bohnen NI, Kaufer DI, Ivanco LS, et al. 2003. Cortical cholinergic function is more severely affected in parkinsonian dementia than in Alzheimer disease: an in vivo positron emission tomographic study. Arch Neurol, 60:1745-8.

Brown RG, Marsden CD. 1984. How common is dementia in Parkinson's disease? Lancet, 2:1262-5.

Burn DJ, Rowan EN, Allan LM, et al. 2006. Motor subtype and cognitive decline in Parkinson's disease, Parkinson's disease with dementia, and dementia with Lewy bodies. J Neurol Neurosurg Psychiatry, 77:585-9.

Chaudhuri KR, Martinez-Martin P, Schapira AH, et al. 2006. International multicenter pilot study of the first comprehensive self-completed nonmotor symptoms questionnaire for Parkinson's disease: the NMSQuest study. Mov Disord, 21:916-23.

Cummings JL. 1988. Intellectual impairment in Parkinson's disease: clinical, pathological, and biochemical correlates. J Geriatr Psychiatry Neurol, 1:24-36.

de Lau LM, Schipper CM, Hofman A, et al. 2005. Prognosis of Parkinson disease: risk of dementia and mortality: the Rotterdam Study. Arch Neurol, 62:1265-9.

Defer GL, Widner H, Marie RM, et al. 1999. Core assessment program for surgical interventional therapies in Parkinson's disease (CAPSIT-PD). Mov Disord, 14:572-84.

Emre M, Aarsland D, Albanese A, et al. 2004. Rivastigmine for dementia associated with Parkinson's disease. New England Journal of Medicine, 351:2509-18.
Enz A, Amstutz R, Bodekke H, et al. 1993. Brain selective inhibition of acetylcholinesterase: a novel approach to therapy for Alzheimer's disease. Prog Brain Res, 98:431-8.

Enz A, Bodekke H, Gray J, et al. 1991. Pharmacologic and clinicopharmacologic properties of SDZ ENA 713, a centrally selective acetylcholinesterase inhibitor. Ann N Y Acad Sci, 640:272-5.

Fernandez HH, Crucian GP, Okun MS, et al. 2005. Mild cognitive impairment in Parkinson's disease: the challenge and the promise. Neuropsychiatric Disease and Treatment, 1:37-50.

Fogelson N, Kogan E, Korczyn AD, et al. 2003. Effects of rivastigmine on the quantitative EEG in demented Parkinsonian patients. Acta Neurol Scand, 107:252-5.

Fuchs GA, Gemende I, Herting B, et al. 2004. Dementia in idiopathic Parkinson's syndrome. J Neurol, 251 Suppl 6:VI/28-32.

Galvin JE, Pollack J, Morris JC. 2006. Clinical phenotype of Parkinson disease dementia. Neurology, 67:1605-11.

Geldmacher DS. 2005. The cost benefit to health plans of pharmacotherapy for Alzheimer's disease. Manag Care, 14:44-6, 49-52, 54-5.

Gibb WR, Lees AJ. 1988. The relevance of the Lewy body to the pathogenesis of idiopathic Parkinson's disease. J Neurol Neurosurg Psychiatry, $51: 745-52$.

Giladi N, Shabtai H, Gurevich T, et al. 2003. Rivastigmine (Exelon) for dementia in patients with Parkinson's disease. Acta Neurol Scand, 108:368-73.

Gurevich TY, Shabtai H, Korczyn AD, et al. 2006. Effect of rivastigmine on tremor in patients with Parkinson's disease and dementia. Mov Disord, 21:1663-6.

Harada CN, Shega JW, Sachs GA. 2005. Rivastigmine for dementia associated with Parkinson's disease. New Engl J Med, 352:1387.

Hely MA, Morris JG, Reid WG, et al. 2005. Sydney Multicenter Study of Parkinson's disease: non-L-dopa-responsive problems dominate at 15 years. Mov Disord, 20:190-9.

Hilker R, Thomas AV, Klein JC, et al. 2005. Dementia in Parkinson disease: functional imaging of cholinergic and dopaminergic pathways. Neurology, 65:1716-22.

Hughes TA, Ross HF, Mindham RH, et al. 2004. Mortality in Parkinson's disease and its association with dementia and depression. Acta Neurol Scand, 110:118-23.

Leroi I, Brandt J, Reich SG, et al. 2004. Randomized placebo-controlled trial of donepezil in cognitive impairment in Parkinson's disease. Int $J$ Geriatr Psychiatry, 19:1-8.

Levy G. 2006. Dementia in Parkinson's disease challenges the "gold standard". Ann Neurol, 58:663-5.

Levy G, Schupf N, Tang MX, et al. 2002. Combined effect of age and severity on the risk of dementia in Parkinson's disease. Ann Neurol, 51:722-9.

Mahler ME, Cummings JL. 1990. Alzheimer disease and the dementia of Parkinson disease: comparative investigations. Alzheimer Dis Assoc Disord, 4:133-49.

Marder KS, Tang MX, Cote L, et al. 1995. The frequency and associated risk factors for dementia in patients with Parkinson's disease. Arch Neurol, 52:695-701.

McKeith I, Del Ser T, Spano P, et al. 2000. Efficacy of rivastigmine in dementia with Lewy bodies: a randomized, double-blind, placebocontrolled international study. Lancet, 356(9247):2031-6.

McKeith IG, Dickson DW, Lowe J, et al. 2005. Diagnosis and management of dementia with Lewy bodies: third report of the DLB Consortium. Neurology, 65:1863-72.

Molchan SE, Vitello B, Minichiello M, et al. 1991. Reciprocal changes in psychosis and mood after physostigmine in a patient with Alzheimer's disease [letter]. Arch Gen Psychiatry, 48:1113.

Miyasaki JM, Shannon K, Voon V, et al. 2006. Practice Parameter: evaluation and treatment of depression, psychosis, and dementia in Parkinson disease (an evidence-based review): report of Quality Standards Subcommittee of the American Academy of Neurology. Neurology, 66:996-1002.

Nasreddine ZS, Phillips NA, Bedirian V, et al. 2005. The Montreal Cognitive Assessment, MoCA: A Brief Screening Tool for Mild Cognitive Impairment. J Am Geriatr Soc, 53:695-9. 
Noe E, Marder K, Bell KL, et al. 2004. Comparison of dementia with Lewy bodies to Alzheimer's disease and Parkinson's disease dementia. Mov Disord, 19:60-7.

Padovani A, Costanzi C, Gilberti N, et al. 2006. Parkinson's disease and dementia. Neurol Sci, 27 Suppl 1:S40-3.

Parkinson J. An essay on the shaking palsy. London, England: Sherwood, Neely, and Jones. p 1817.

Perl DP, Olanow CW, Calne D. 1998. Alzheimer's disease and Parkinson's disease: distinct entities or extremes of a spectrum of neurodegeneration? Ann Neurol, 44(3 Suppl 1):S19-31.

Pillon B. 2002. Neuropsychological assessment for management of patients with deep brain stimulation. Mov Disord, 17 Suppl 3:S116-22.

Poewe W, Wolters E, Emre M, et al. 2006. Long-term benefits of rivastigmine in dementia associated with Parkinson's disease: an active treatment extension study. Mov Disord, 21:456-61.

Ravina B, Putt M, Siderowf A, et al. 2005. Donepezil for dementia in Parkinson's disease: a randomised, double blind, placebo controlled, crossover study. J Neurol Neurosurg Psychiatry, 76:934-9.

Reading PJ, Luce AK, McKeith IG. 2001. Rivastigmine in the treatment of Parkinsonian psychosis and cognitive impairment: preliminary findings from an open trial. Mov Disord, 16:1171-4.
Richard IH, Justus AW, Greig NH, et al. 2002. Worsening of motor function and mood in a patient with Parkinson's disease after pharmacologic challenge with oral rivastigmine. Clin Neuropharm, 25:296-9.

Rippon GA, Marder KS. 2005. Dementia in Parkinson's disease. Adv Neurol, 96:95-113.

Saint-Cyr JA, Trepanier LL, Kumar R, et al. 2000. Neuropsychological consequences of chronic bilateral stimulation of the subthalamic nucleus in Parkinson's disease. Brain, 123:2091-108.

Voon V, Kubu C, Krack P, et al. 2006. Deep brain stimulation: neuropsychological and neuropsychiatric issues. Mov Disord, 14:S305-27.

Wesnes KA, McKeith I, Edgar C, et al. 2005. Benefits of rivastigmine on attention in dementia associated with Parkinson's disease. Neurology, 65:1654-6.

Whitehouse P. 1987. Clinical and neurochemical consequences of neuronal loss in the nucleus basalis of Meynert in Parkinson's disease and Alzheimer's disease. Adv Neurol, 45:393-7.

Willan AR, Goeree R, Pullenayegum EM, et al. 2006. Economic evaluation of rivastigmine in patients with Parkinson's disease dementia. Pharmacoeconomics, 24:93-106. 
